\title{
INFLUENCIA DE LAS TIC EN LA UTILIZACIÓN DE MATERIALES Y RECURSOS EN LOS PROCESOS DE ENSEÑANZA-APRENDIZAJE DE LA UNIVERSIDAD DE LLEIDA: ¿USO O ABUSO?
}

\author{
(INFLUENCE OF ICT IN THE USE OF MATERIALS AND RESOURCES IN THE TEACHING AND \\ LEARNING AT THE UNIVERSITY OF LLEIDA: USE OR ABUSE?)
}

\author{
Óscar Flores Alarcia \\ Isabel del Arco Bravo \\ Universidad de Lleida (España)
}

\section{RESUMEN}

El artículo es un estudio realizado con el objetivo de observar la utilización de recursos de aprendizaje en diferentes asignaturas presenciales, semipresenciales y no presenciales de la Universidad de Lleida. La investigación se desarrolló bajo la modalidad de estudio de casos, recogiendo datos de profesorado y estudiantado a través de análisis documental, cuestionarios y entrevistas. El estudio mostró que, independientemente de la modalidad formativa de las asignaturas, se utilizaba una variedad considerable de recursos para hacer llegar la información a los estudiantes, aunque se detectó una baja mediación entre los materiales que el profesorado publicaba y el acceso a estos por parte de los estudiantes. Consideramos que todavía queda camino por recorrer en el aprovechamiento de los recursos TIC en los procesos formativos universitarios.

Palabras clave: tecnologías de la información y la comunicación, enseñanza-aprendizaje, educación superior, recursos materiales.

\begin{abstract}
The article is a report on a study done in order to observe the use of learning resources in different face-to-face, blended and online courses at the University of Lleida. The research was conducted in the form of case studies in which data was collected from faculty and students through documentary analysis, questionnaires, and interviews. The study showed that teachers used a considerable variety of resources to deliver information to students, although we did, however, detect a low correlation between faculty published materials and access to those materials for students. We believe that there is still room for improvement in the use of ICT resources in the university training processes.
\end{abstract}

Keywords: information and communication technologies, teaching and learning, higher education, material resources. 
Es indudable que el e-learning, referido al "desarrollo de experiencias formativas no presenciales de carácter interactivo, abierto y flexible, que tienen en las TIC, y principalmente en la red Internet, el soporte fundamental para su desarrollo" (Baelo, 2009, p. 88), ha abierto nuevas posibilidades formativas en el sistema educativo en general, y en la universidad en particular.

Desde nuestro punto de vista, las TIC pueden aportar un gran abanico de posibilidades en los procesos de enseñanza-aprendizaje. Izquierdo y María (2010) realizan un trabajo profundo de análisis del impacto de las TIC en la transformación de la enseñanza universitaria y de la necesidad de repensar los modelos de enseñanza y aprendizaje.

Este modelo necesariamente debe comportar cambios en los modos de enseñar y aprender (Escofet, Alabart y Vilà, 2006, p. 11):

- Del profesor como fuente del conocimiento al facilitador del aprendizaje.

- Del alumno receptor al alumno constructor.

- Del individuo aislado al grupo que colabora.

- Del uso de las tecnologías para la enseñanza al uso de las TIC para el aprendizaje.

El uso de las TIC en un proceso formativo, sobre todo si este se desarrolla bajo la modalidad semipresencial o $100 \%$ no presencial, debe implicar necesariamente tres manifestaciones que se pueden considerar como una respuesta práctica a los cambios en la educación superior debido de la influencia de las TIC (Salinas, 2004, p. 6):

- Cambios en el rol del profesor. La institución educativa y el profesor dejan de ser fuentes de todo conocimiento, y el profesor debe pasar a actuar como guía de los alumnos y facilitarles el uso de los recursos y las herramientas que necesitan para explorar y elaborar nuevos conocimientos y destrezas.

- Cambios en el rol del alumnado. El contacto de los alumnos con las TIC requerirá acciones educativas relacionadas con el uso, selección, utilización y organización de la información, de manera que el alumno vaya formándose como un maduro ciudadano de la sociedad de la información. 
- Cambios en la metodología. La utilización de las redes para la enseñanza puede permitir el desarrollo de nuevas configuraciones formativas que superen las deficiencias de los sistemas convencionales, ya sean presenciales o a distancia.

Plantearse un cambio en los roles de profesorado y estudiantado y de las metodologías, dejar de pensar en el procedimiento "transmitir conocimientos" para pasar a un nuevo modelo donde el alumno debe descubrir y construir su conocimiento con la ayuda de los compañeros y del profesor nos lleva a considerar que los modelos tradicionales de evaluación, basados sobre todo en la realización de un examen final, en que se muestren los conocimientos teóricos adquiridos por los alumnos, no responderán a saber exactamente qué se ha aprendido.

Monge (2007, p. 283) expresa el estereotipo que aún predomina en el sistema educativo: "el objetivo del acto educativo se ve como un temario para impartir y no como un conjunto de procedimientos, actitudes y conceptos para alcanzar por parte del estudiante. Los sistemas de evaluación están diseñados a menudo para medir únicamente los conocimientos teóricos de los alumnos y no sus capacidades para hacer. Las clases se organizan de manera que el docente habla y al alumno se le "invita" a escuchar, aunque se intenta que participe en la clase a través de preguntas".

Según este autor, los roles tradicionales profesor-alumno han quedado obsoletos y hay que poner en tela de juicio el proceso de aprendizaje entendido como la transmisión de conocimiento, y buscar nuevos marcos teóricos para explicar el proceso de enseñanza-aprendizaje. Considera este autor que la búsqueda de nuevos modelos para desarrollar el acto formativo debe desembocar en nuevos planteamientos para la evaluación: "todo esto apunta la inoperatividad de los sistemas de evaluación actuales, que están basados en la memorización” (Monge, 2007, p. 34).

En la misma línea que Monge se expresan Pedró y Benavides (2007). Estos autores reconocen que las TIC son vistas por los docentes como herramientas adecuadas para cubrir algunas asignaturas pendientes, como la renovación de contenidos y el sistema de evaluación, frente a un currículo tradicional donde el conocimiento se sigue acumulando de forma "enciclopédica", con un currículum poco flexible, y donde el conocimiento se organiza todavía en asignaturas y grados. Es con esta renovación de contenidos y planteándose nuevos sistemas de evaluación que consideran estos autores que realmente se podrá proveer a los alumnos de estrategias de análisis y resolución de problemas indispensables hoy en día. 
La aparición de las tecnologías de la información ha permitido incorporar a más objetos de aprendizaje en el proceso formativo. Como indican Mülbert y Matuzawapara (2011), encontrar el equilibrio en la forma de presentar el contenido y el concepto que se desea transmitir es un gran desafío, ya que a través de las TIC podemos captar la atención de los estudiantes con estímulos que van más allá de la visión (para la lectura de texto estático para el caso del material impreso): la audición (la narración y efectos de sonido en general), la interacción con el contenido (el usuario puede elegir la opción que desee profundizar en su estudio), y la lectura textual con animación (gráficos animados).

A pesar de las potencialidades de las TIC en la formación, la realidad no siempre es tan prometedora como parece. El uso cada vez más generalizado de Internet no tiene por qué implicar la modificación de prácticas ni de nuevas dimensiones de aprendizaje. Excepto en algunas ocasiones, el uso de las TIC sigue tendiendo a imponer un modelo formativo centrado en los contenidos, traspasando lo que se hace presencialmente en la red. Ante esta situación, el efecto que se produce es lo que estos autores denominan "navegación por apuntes": "los estudiantes acceden a los apuntes de sus profesores a través de la red sin que haya más mediación o valor añadido en el proceso de enseñanza-aprendizaje. Se trata de utilizar la tecnología para hacer lo mismo" (Gros y Kirschner, 2006, p. 12).

Mahdizadeh, Biemans y Mulder (2008) desarrollaron un estudio en Holanda para conocer los principales usos de los entornos tecnológicos en la educación superior por parte de los profesores. En la tabla 1 se muestra un resumen de los principales resultados obtenidos sobre los usos que realizan los profesores del entorno e-learning en el desarrollo de la docencia:

\begin{tabular}{|c|c|}
\hline Principales usos & Tareas menos realizadas \\
\hline $\begin{array}{ll}\text { - } & \text { Presentar los materiales del curso y la } \\
\text { - } & \text { literatura. } \\
\text { - } & \text { Colgar presentaciones de PowerPoint. } \\
\text { - } & \text { Información sobre el curso. }\end{array}$ & $\begin{array}{ll}\text { - } & \text { Test online. } \\
\text { - } & \text { Videoconferencia y encuentros en red. } \\
\text { - } & \text { Pizarras compartidas. } \\
\text { - } & \text { Conferencias de voz. }\end{array}$ \\
\hline
\end{tabular}

Tabla 1. Usos profesorado entorno e-learning. Adaptado de Mahdizadeh, Biemans y Mulder, 20o8, p. 147

Estos autores también indagaron sobre la opinión del profesorado en cuanto a qué valor añadido para el aprendizaje de los estudiantes ofrece la utilización de 
entornos online. En la tabla 2 se muestra un resumen de los aspectos más valorados y los menos valorados:

\begin{tabular}{|c|c|}
\hline Aspectos más valorados & Aspectos menos valorados \\
\hline $\begin{array}{ll}\text { - } & \text { Presentación de materiales del curso y } \\
\text { - } & \text { de la literatura. } \\
\text { - } & \text { Presentaciones de PowerPoint. } \\
\text { - } & \text { Correo electrónico. }\end{array}$ & $\begin{array}{ll}\text { - } & \text { Discusiones online. } \\
\text { - } & \text { Test online. } \\
\text { - } & \text { Videoconferencia y encuentros en red. } \\
\text { - } & \text { Pizarras compartidas. } \\
\text { - } & \text { Conferencias de voz. }\end{array}$ \\
\hline
\end{tabular}

Tabla 2. Valor añadido entornos Online. Adaptado de Mahdizadeh, Biemans y Mulder, 20o8, p. 147

En cuanto a otra parte del estudio, en el que se recogieron opiniones de los profesores sobre los entornos de aprendizaje y sobre impedimentos a tener en cuenta con respecto al uso de las TIC en la docencia (Mahdizadeh, Biemans y Mulder, 2008, p. 148), la mayoría se mostraron conformes con la idea de que la calidad del aprendizaje de los estudiantes mejora con el uso de las computadoras y de Internet, aunque también mostraron un alto grado de acuerdo en la falta de programas y sitios web que les ofrezcan un adecuado apoyo a las tareas docentes. Otro resultado a destacar es el hecho de que los profesores se mostraron totalmente en desacuerdo con la idea de que los entornos tecnológicos no tienen un valor añadido en sus cursos, aunque la mayoría seguía prefiriendo la docencia presencial.

Por su parte, Chen (2007) realizó un estudio para observar la valoración de los estudiantes en un curso online intensivo combinando estrategias de enseñanza conductistas y constructivistas. Sobre las actitudes y opiniones de los estudiantes referentes a la experiencia del curso, los resultados mostraron que estos quedaron altamente satisfechos con la calidad, el contenido y el diseño del curso, los métodos instruccionales utilizados y las experiencias de aprendizaje y la colaboración en equipo. En cuanto a cómo el diseño del curso impactó en el aprendizaje de los estudiantes, todos indicaron que las tareas de aprendizaje eran prácticas y aplicables. Asimismo, en general los estudiantes mostraron satisfacción con el apoyo tecnológico ofrecido, e incluso cambió su punto de vista sobre la aplicación de la tecnología de Internet a la educación. En cuanto a los contenidos y el apoyo en el aprendizaje, la mayoría indicaron que el curso era práctico e informativo, y que la estructura del curso estaba bien organizada y el feedback del formador contribuía a la mejora en el aprendizaje. Finalmente, sobre el apoyo social los estudiantes indicaron que la 
colaboración e interacción no solo beneficiaban su aprendizaje, sino también su experiencia personal.

En la importancia de los recursos y los materiales en el proceso formativo, sobretodo cuando las TIC entran en juego, también destaca Wright (2003), cuando, entre otros criterios para valorar la calidad de un curso, indica que es muy importante que el material esté bien organizado y que la secuenciación de los contenidos sea adecuada. También destaca este autor el hecho de que los recursos y los materiales deben ser atractivos, apropiados para los contenidos que se trabajan y adecuados a las características de los estudiantes a quienes se dirige. Finalmente, también debe tenerse en cuenta la importancia de que los recursos sean fácilmente accesibles parar los estudiantes.

En cualquier caso, los cambios que la docencia universitaria requiere para adaptarse al desarrollo de estrategias formativas utilizando las TIC, pasarán, de acuerdo con Marín y Romero (2009, p. 102), por un replanteamiento del proceso que se apoye en el "aprendizaje haciendo" en lugar de la concepción de la "enseñanza hablando".

Desde esta perspectiva relativa a la necesidad de replantearse el proceso formativo cuando las tecnologías entran a formar parte del mismo, se realizó una investigación en la Universidad de Lleida con el objetivo de analizar el uso de las TIC en el desarrollo de los procesos de enseñanza y aprendizaje.

La investigación, realizada durante los cursos 2007-2008, 2008-2009 y 20092010, se centró en observar la utilización de los recursos y materiales de aprendizaje en diferentes asignaturas de la universidad en función de si estas se desarrollaban bajo la modalidad presencial, semipresencial o no presencial. Para el estudio se recogieron datos tanto del profesorado como del estudiantado.

\section{METODOLOGÍA}

Este trabajo se enmarca en una perspectiva cualitativa o humanísticointerpretativa y crítica (Hernández Pina, 1995), con la pretensión de describir e interpretar los fenómenos sociales y estudiar los significados e intenciones de las acciones humanas desde la perspectiva de los propios agentes sociales (Latorre, del Rincón, Arnal, 2003, p. 199). Por las características del contexto en que se sitúa el trabajo de investigación (se centra en una realidad concreta, la Universidad de 
Lleida) y los objetivos que se plantean, el estudio se enmarca como un estudio de casos.

El diseño metodológico se articuló alrededor de tres técnicas de recogida de datos. Primeramente se realizaría un análisis de las planificaciones docentes de aquellas asignaturas que serían objeto de estudio. Este hecho nos permitiría, por un lado, acercarnos al proceso formativo antes de su desarrollo y, por otro, indagar de qué manera la utilización de las TIC influencia en los diferentes aspectos que planifica el profesorado para llevar a cabo el proceso formativo. Para el análisis documental de los programas de las asignaturas, uno de los problemas que nos encontramos fue qué criterio seguir para establecer un modelo que nos sirviera de guía para elaborar un registro homogéneo. La solución a este dilema nos la ofreció una herramienta que la propia Universidad de Lleida ha diseñado para planificar las asignaturas. Esta herramienta se llama Tabla de Planificación Docente, un aplicativo común en todas las asignaturas de la universidad que permite a cada profesor planificar la docencia siguiendo unas directrices establecidas.

Además del análisis documental, consideramos que era necesario, para ampliar la aproximación a la realidad objeto de estudio, desarrollar alguna técnica que nos permitiera llegar a un gran número de informantes, tanto del profesorado como del estudiantado. Para esta finalidad utilizaríamos los cuestionarios. Para el diseño de los mismos se desarrolló un proceso de creación y validación de la herramienta. En primer lugar, para elaborar la primera versión de la herramienta, se agruparon los elementos sobre los que se pretendía recoger datos y se elaboraron las cuestiones sobre las que se debían articular las preguntas. Una vez diseñada la primera versión del mismo, se consideró necesario contar con tres perfiles diferentes de jueces para la validación: expertos y expertas en el ámbito de la utilización de las TIC en procesos de enseñanza-aprendizaje, profesorado universitario y estudiantes. Los elementos que se consideró necesario evaluar, para cada ítem, fueron la univocidad y la adecuación de estos. Una vez realizado este trabajo de validación, se procedió al análisis cuantitativo y cualitativo de las aportaciones de los jueces, que nos permitió realizar la versión final de la herramienta.

Finalmente, se consideró pertinente elegir una tercera técnica que nos permitiera acercarnos a los elementos objeto de estudio pudiendo recoger percepciones y sensaciones de los informantes una vez finalizado el proceso formativo. Por este motivo, la tercera técnica utilizada fue la entrevista. Para diseñar la herramienta, se diseñó un protocolo para la recogida de datos de la entrevista que se validó con 
la colaboración de un profesor experto en investigación utilizando metodología cualitativa.

\section{Fuentes de información}

El criterio principal para acceder a las fuentes de información fue localizar asignaturas presenciales, semipresenciales y no presenciales de la Universidad de Lleida. Para ello, se pudo acceder a los datos de que dispone el Área de Soporte a la Innovación Docente y E-learning, una unidad estructural del Instituto de Ciencias de la Educación -Centro de Formación Continua dedicada a dar apoyo pedagógico e informático al profesorado para incorporar las TIC en los procesos formativos-. A partir de los datos disponibles se seleccionaron un total de 29 asignaturas: nueve presenciales, diez semipresenciales y diez no presenciales.

Una vez elegidas estas asignaturas se inició el proceso para disponer de las planificaciones de estas para realizar el análisis documental. Algunas se encontraron a través de la misma Web de la universidad, otras a través del acceso al programa colgado en el campus virtual.

Para realizar la recogida de datos del profesorado a través del cuestionario se partió de las 29 asignaturas elegidas previamente para realizar el análisis documental. A partir de esta masa de profesorado se inició un proceso de búsqueda de otros docentes de las diferentes facultades y escuelas. El criterio seguía siendo el mismo: localizar procesos formativos presenciales, semipresenciales y no presenciales en diferentes ámbitos.

Para ello se accedió a las páginas Web de las diferentes facultades o escuelas y se revisaron los directorios que estas ofrecen con los datos del personal docente. En algunos casos se optó por seleccionar profesorado de manera aleatoria sin conocer en qué modalidad formativa desarrollaban sus asignaturas. En otros, se escogió personal que sabíamos, gracias a la información que nos proporcionó el Área de Apoyo a la Innovación Docente y E-learning, que desarrollaban algún proceso formativo utilizando las TIC.

Realizando este procedimiento se fue incorporando profesorado en una base de datos hasta que acumulamos un total de 212 individuos, de los cuales se recibió respuesta de 71,43 hombresy 28 mujeres (6o,6\% y $39,4 \%$, respectivamente). En cuanto a la modalidad formativa, 39 de los 71 casos (el $54,9 \%$ ) respondieron el cuestionario 
por asignaturas totalmente presenciales, $27(38,7 \%)$ por semipresenciales y cinco (un $7,0 \%$ ) por asignaturas totalmente virtuales.

Para la recogida de datos del estudiantado a través del cuestionario se partió también de las 29 asignaturas que se seleccionaron inicialmente para realizar el análisis documental. Se contactó con el profesorado responsable de las asignaturas y se pidió su permiso para poder acudir un día determinado en el aula y realizar la recogida de datos. Finalmente se obtuvieron 658 respuestas, 163 (un 24,8\%) pertenecían a hombres y 478 (un 72,6\%) a mujeres (17 no respondieron este ítem). En cuanto a la modalidad formativa, 405 (un 61,6\%) respondieron por la realización de asignaturas totalmente presenciales, 181 (un 27,5\%) por semipresenciales y 67 (un 10,2\%) por asignaturas totalmente no presenciales (cinco individuos no respondieron este ítem).

Para finalizar este apartado referente a las fuentes de información cabe mencionar el acceso a estas para realizar las entrevistas. En este caso se partió de las 29 asignaturas iniciales que se seleccionaron para realizar el análisis documental, pero también se aprovechó la base de datos de profesores que se recopiló para realizar la recogida de datos a través del cuestionario. Finalmente, se contactó con doce profesores y profesoras de diferentes escuelas y facultades de la UdL. De este grupo de doce cuatro desarrollan asignaturas presenciales, cuatro asignaturas semipresenciales, y cuatro más, asignaturas no presenciales.

\section{RESULTADOS}

\section{Análisis de las planificaciones}

En las planificaciones de las asignaturas presenciales no se explicitaba claramente los materiales que se utilizarían durante la impartición de la asignatura. En ninguna de estas planificaciones aparecía la utilización de materiales multimedia interactivos. Solo se observó una en la que se explicitaba el uso de soporte audiovisual a las explicaciones. Artículos, libros y vídeos son la única referencia que encontramos de materiales propiamente dichos. En la mayoría de planificaciones se hacía más referencia al lugar donde se trabajaría (el aula, laboratorios, aulas de informática...) que el material que se utilizaría. Resulta interesante comentar que en una planificación se hacía mención al uso del campus virtual para entregar actividades. 
Las planificaciones de las asignaturas semipresenciales mostraron que los textos interactivos y materiales multimedia eran los materiales más utilizados (cinco planificaciones explicitaban esta tipología de materiales). También se observó que aparecía el término "apuntes" como material de la asignatura. Por otro lado, aparecían materiales de carácter más textual (apuntes, artículos, referencias bibliográficas, dossier de casos, material teórico...). Aparte de una planificación que no explicitaba la tipología de materiales que se utilizarían, en dos planificaciones había listas de problemas y pruebas y exámenes online. Aunque no pueden considerarse materiales propiamente dichos, también hay que mencionar que en algunas de las planificaciones se hacía mención al uso de laboratorios y de aulas de informática para el desarrollo de la docencia.

Respecto a las asignaturas no presenciales, en general, la mayoría hacían referencia a la utilización de materiales interactivos y en formato Web (textos multimedia, páginas Web, imágenes, enlaces...). En cuatro programas se citaba explícitamente el uso de apuntes, libros, capítulos de un libro y recursos bibliográficos como materiales de la asignatura. Una planificación hacía referencia a la utilización de novelas y películas como material formativo, y solo un programa citaba la utilización de material autoformativo para los estudiantes. Finalmente, cabe comentar que en dos planificaciones no se explicitaba ningún apartado que hiciera referencia a materiales de la asignatura.

\section{Cuestionarios}

A través del cuestionario se preguntó al profesorado y estudiantado la frecuencia de uso de los siguientes recursos y materiales de aprendizaje: apuntes o esquemas de contenidos elaborados por él mismo, presentaciones a través de diapositivas de clase (con transparencias, PowerPoint...), guías o pautas para realizar ejercicios, prácticas..., trabajos de los estudiantes (de otros cursos o actuales), exámenes anteriores, artículos de revistas, capítulos o partes de libros, material gráfico (diagramas, esquemas, dibujos y/o fotografías,...), vídeos, audio, animaciones y/o simulaciones, bases de datos, CD interactivo, páginas Web. Las respuestas obtenidas fueron agrupadas según la modalidad formativa de la asignatura, el género de los informantes y por experiencia de estos en la universidad.

En referencia a la modalidad formativa (tablas 3 y 4), el comportamiento de los datos en las respuestas del profesorado fue muy similar en las asignaturas presenciales y semipresenciales. En ambos casos los apuntes (la suma de los que responden que siempre o a menudo utilizan esta estrategia da el $87,2 \%$ en las 
presenciales y el $88,9 \%$ en las semipresenciales), las presentaciones a través de diapositivas $(79,5 \%$ y $88,9 \%$, respectivamente) y las guías para realizar ejercicios $(69,3 \%$ y $88,8 \%$ respectivamente) eran los recursos más utilizados. En el resto de recursos y materiales el nivel de uso iba disminuyendo de una manera similar en las asignaturas presenciales y semipresenciales. Cabe destacar que en el uso de páginas Web en las asignaturas presenciales la opción "siempre" presentaba un resultado significativamente inferior respecto a la media. En cuanto a las opciones que podríamos llamar más interactivas -como el uso de vídeos, audio, animaciones, CD...- no se observó un aumento, ni en las asignaturas semipresenciales ni en las virtuales. En cambio, en las asignaturas virtuales destacaba el uso de material gráfico y capítulos o partes de libros. 
O. FLORES; I. DEL ARCO

INFLUENCIA DE LAS TIC EN LA UTILIZACIÓN DE MATERIALES Y RECURSOS EN LOS PROCESOS DE ENSEÑANZA-APRENDIZAJE...

\begin{tabular}{|c|c|c|c|c|c|c|}
\hline \multirow{3}{*}{ 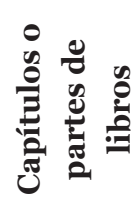 } & $\vec{z}$ & ฉิ & \& & શ્స & & ' \\
\hline & $\bar{s}$ & $\vec{F}$ & $\begin{array}{l}10 \\
\infty \\
\infty\end{array}$ & $\begin{array}{c}\hat{\sigma} \\
\hat{N}\end{array}$ & $\begin{array}{l}\text { N } \\
\text { ลิ }\end{array}$ & $\begin{array}{l}\text { N } \\
\text { సิ }\end{array}$ \\
\hline & A & $\hat{\curvearrowright}$ & $\begin{array}{l}N \\
\infty \\
\text { ô }\end{array}$ & $\begin{array}{l}\text { No } \\
\text { ô } \\
\text { N }\end{array}$ & ث̊ & $\hat{\wedge}$ \\
\hline \multirow{3}{*}{ 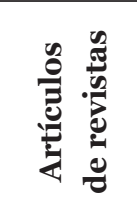 } & $\hat{\mathbf{z}}$ & 오 & \& & o & 1 & ' \\
\hline & $\bar{\mho}$ & $\begin{array}{l}\infty \\
\underset{J}{f}\end{array}$ & $\begin{array}{l}\text { ñ } \\
\text { ஸి }\end{array}$ & $\begin{array}{l}\text { N } \\
\text { ลิ }\end{array}$ & $\begin{array}{c}\hat{\sigma} \\
\hat{N}\end{array}$ & ๙ิ \\
\hline & $\theta$ & $\begin{array}{l}\infty \\
\text { త̂ }\end{array}$ & $\begin{array}{l}0 \\
\text { L } \\
\text { No }\end{array}$ & $\stackrel{\curvearrowright}{\approx}$ & $\hat{\text { సิ }}$ & $\begin{array}{l}\infty \\
\text { ज̂ }\end{array}$ \\
\hline \multirow{3}{*}{ 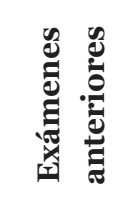 } & $\hat{\mathbf{z}}$ & ' & ' & શે & ' & $\infty$ \\
\hline & $\bar{\mho}$ & 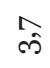 & $\stackrel{+}{N}$ & $\begin{array}{l}\text { مิ } \\
\text { הิ }\end{array}$ & $\begin{array}{l}0 \\
\text { an }\end{array}$ & ભి \\
\hline & $\theta$ & $\overrightarrow{10}$ & $\begin{array}{l}\text { na } \\
0\end{array}$ & $\begin{array}{l}+ \\
\stackrel{0}{0}\end{array}$ & $\begin{array}{l}12 \\
\stackrel{2}{\text { in }}\end{array}$ & $F$ \\
\hline \multirow{3}{*}{ ש̊ } & $\vec{z}$ & 8 & ' & ' & ' & fo \\
\hline & $\bar{\mho}$ & $\begin{array}{l}\hat{\sigma} \\
\text { s. }\end{array}$ & $\begin{array}{l}\text { N } \\
\text { ลิ }\end{array}$ & $\begin{array}{l}10 \\
\infty \\
0\end{array}$ & $\begin{array}{l}\infty \\
\underset{J}{f}\end{array}$ & $\begin{array}{l}12 \\
\infty 0 \\
\end{array}$ \\
\hline & a & $\begin{array}{l}\infty \\
\text { Nิ }\end{array}$ & $\stackrel{\overbrace{}}{\stackrel{\sigma}{\sim}}$ & $\begin{array}{l}10 \\
\stackrel{\text { N }}{2}\end{array}$ & $\hat{\text { กิ }}$ & ڤి \\
\hline \multirow{3}{*}{ 莺 } & $\hat{\mathbf{z}}$ & $\infty$ & ' & 1 & ' & ' \\
\hline & $\bar{s}$ & $\vec{\infty}$ & $\hat{o}$ & $\Rightarrow$ & ' & ' \\
\hline & $A$ & $\begin{array}{l}\infty \\
0 \\
m\end{array}$ & $\begin{array}{l}10 \\
\infty \\
\infty \\
\infty\end{array}$ & $\begin{array}{c}N \\
\text { No } \\
\text { N }\end{array}$ & I & ' \\
\hline \multirow{3}{*}{ 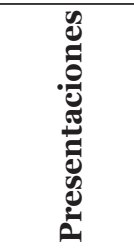 } & $\overrightarrow{\mathbf{z}}$ & 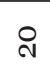 & ' & ' & ' & 8 \\
\hline & है & $\begin{array}{l}0 \\
\text { L } \\
10\end{array}$ & 饣ి & $\stackrel{t}{N}$ & के & ' \\
\hline & $\theta$ & n & $\begin{array}{l}N \\
\text { ô } \\
\text { N }\end{array}$ & $\stackrel{\curvearrowright}{\hat{\sim}}$ & I & $\begin{array}{l}0 \\
\text { iv }\end{array}$ \\
\hline \multirow{3}{*}{$\frac{\stackrel{0}{\Xi}}{3}$} & $\vec{z}$ & $\infty$ & ' & ' & 요 & ' \\
\hline & $\tilde{\varkappa}$ & $\stackrel{a}{i}$ & مิ & 1 & $\overrightarrow{=}$ & I \\
\hline & $A$ & $\begin{array}{l}+ \\
6 \\
10\end{array}$ & $\begin{array}{l}\infty \\
\hat{\infty} \\
\hat{m}\end{array}$ & 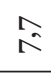 & I & 1 \\
\hline & & हैं & 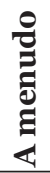 & 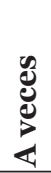 & : & $\begin{array}{l}\text { J్ } \\
\Xi \\
Z\end{array}$ \\
\hline
\end{tabular}

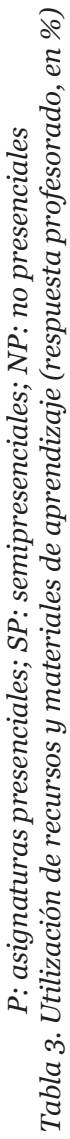


O. Flores; I. DEL ARCO

INFLUENCIA DE LAS TIC EN LA UTILIZACIÓN DE MATERIALES Y RECURSOS EN LOS PROCESOS DE ENSEÑANZA-APRENDIZAJE...

\begin{tabular}{|c|c|c|c|c|c|c|}
\hline \multirow{3}{*}{ 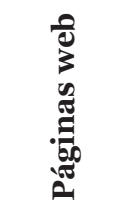 } & 点 & คे & ลั & 우 & ' & ' \\
\hline & जे & $\begin{array}{l}0 \\
\text { in }\end{array}$ & $\begin{array}{l}\text { యे } \\
\text { ले }\end{array}$ & $\begin{array}{l}\text { V } \\
\text { ลิ }\end{array}$ & $\stackrel{\nabla}{\sim}$ & के \\
\hline & A & $\hat{\sim}$ & $\hat{\mathfrak{N}}$ & $\begin{array}{l}\infty \\
\text { on } \\
\text { in }\end{array}$ & $\stackrel{\curvearrowright}{\hat{\sigma}}$ & Lิ \\
\hline \multirow{3}{*}{ อิ } & $\overrightarrow{\mathrm{z}}$ & ' & ' & 1 & ลे & $\infty$ \\
\hline & 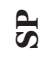 & ஸे & ஸิ & ஸิ & $\begin{array}{l}\text { N } \\
\text { ลิ }\end{array}$ & $\hat{b}$ \\
\hline & $A$ & I & $\begin{array}{l}0 \\
\text { N }\end{array}$ & $\begin{array}{l}\infty \\
\text { Î } \\
\text { In }\end{array}$ & $\stackrel{\vartheta}{\hat{\sigma}}$ & ฉે \\
\hline \multirow{3}{*}{ 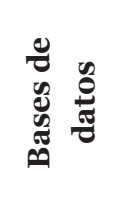 } & $\hat{z}$ & ㅇ & ' & ' & $\stackrel{\text { N }}{ }$ & 8 \\
\hline & $\tilde{\xi}$ & $\stackrel{+}{N}$ & $=$ & $\begin{array}{l}\infty \\
\underset{J}{f}\end{array}$ & 오 & $\hat{o}$ \\
\hline & $A$ & ' & $\stackrel{2}{\stackrel{2}{0}}$ & ๙ิ) & 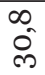 & $\begin{array}{l}N \\
\text { No } \\
\text { No }\end{array}$ \\
\hline \multirow{3}{*}{ : } & 文 & ì & ' & ' & 1 & $\infty$ \\
\hline & $\tilde{\xi}$ & 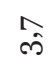 & $\stackrel{\nabla}{\sim}$ & $\begin{array}{l}\text { V } \\
\text { ลิ }\end{array}$ & $\begin{array}{l}\text { N } \\
\text { ลี }\end{array}$ & $\hat{\circ}$ \\
\hline & $a$ & $\hat{\curvearrowright}$ & î & $\begin{array}{l}{ }_{2}^{2} \\
0\end{array}$ & $\stackrel{\vartheta}{\hat{\sigma}}$ & 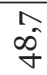 \\
\hline \multirow{3}{*}{$\frac{\mathscr{2}}{3}$} & $\frac{\theta}{z}$ & & ลे & ลे & ㅇํ & $\stackrel{\circ}{+}$ \\
\hline & जे & $\hat{\text { ஸे }}$ & 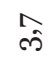 & लิ & $\begin{array}{l}\text { N } \\
\text { ปิ }\end{array}$ & $\hat{\theta}$ \\
\hline & $A$ & है & $\begin{array}{l}0 \\
\text { in }\end{array}$ & $\hat{\curvearrowright}$ & ثئ & to \\
\hline \multirow{3}{*}{$\overbrace{0}^{0}$} & 㝋 & ' & 오 & fo & ' & $\stackrel{\circ}{+}$ \\
\hline & $\bar{\sigma}$ & $\stackrel{⿱}{N}$ & $\begin{array}{l}10 \\
\infty \\
\infty\end{array}$ & $\hat{o}$ & $\vec{F}$ & $\begin{array}{l}\text { N } \\
\text { సิ }\end{array}$ \\
\hline & $A$ & $\begin{array}{l}\text { 20 } \\
\stackrel{-}{1}\end{array}$ & $\begin{array}{l}2 \\
0^{2}\end{array}$ & $\begin{array}{l}0 \\
\text { î } \\
\text { N }\end{array}$ & 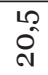 & 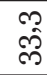 \\
\hline \multirow{4}{*}{ م } & $\frac{\theta}{z}$ & f & ลิ & ' & ' & $\stackrel{P}{q}$ \\
\hline & $\vec{v}$ & $\begin{array}{l}0 \\
\text { an }\end{array}$ & $\begin{array}{l}0 \\
\text { an }\end{array}$ & $\begin{array}{l}10 \\
\infty \\
\infty\end{array}$ & $\begin{array}{l}L^{2} \\
\infty^{2}\end{array}$ & ' \\
\hline & A & $\begin{array}{l}\infty \\
\text { Oे } \\
\text { en }\end{array}$ & $\begin{array}{l}\infty \\
\hat{\infty}^{-}\end{array}$ & $\stackrel{\curvearrowright}{\stackrel{\Omega}{\sim}}$ & $\begin{array}{l}2 \\
0^{2}\end{array}$ & เి \\
\hline & & 总 & 욜 & 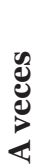 & $\stackrel{8}{0}$ & $\begin{array}{l}\text { J } \\
\text { ב } \\
\text { Z }\end{array}$ \\
\hline
\end{tabular}


En cuanto a las respuestas del estudiantado según la modalidad formativa (tablas 5 y 6), comparándolas con las respuestas del profesorado, se pudo observar que coincidía la opinión de que los apuntes, las presentaciones a través de diapositivas (en el caso de las presentaciones, resulta interesante observar la elevada respuesta de los estudiantes de asignaturas presenciales y semipresenciales sobre el uso de estas, más de un 70\% sumando "siempre" y "a menudo") y las guías para realizar ejercicios eran las estrategias más utilizadas. Es curioso observar cómo los estudiantes de las asignaturas virtuales no consideraban "apuntes" los materiales de consulta que deja el profesor (solo un $3 \%$ de estos respondieron que el profesor siempre usa apuntes y un $16,4 \%$ responden la opción "a menudo", ambos resultados significativamente inferiores a la media). Sobre el resto de recursos y materiales, los estudiantes coincidieron en que eran poco utilizados, excepto la opción "páginas Web", en que la respuesta de los estudiantes de asignaturas virtuales fue significativamente superior en las opciones "siempre" o "a menudo" ( 40,3 y 38,8\%, respectivamente). Probablemente, los estudiantes consideraban las páginas Web como los materiales de consulta que los profesores cuelgan en el campus virtual. 
O. FLORES; I. DEL ARCO

INFLUENCIA DE LAS TIC EN LA UTILIZACIÓN DE MATERIALES Y RECURSOS EN LOS PROCESOS DE ENSEÑANZA-APRENDIZAJE...

\begin{tabular}{|c|c|c|c|c|c|c|}
\hline \multirow{3}{*}{ 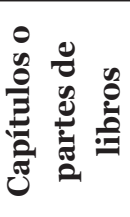 } & 完 & ๙ิ ๙ิ & $\stackrel{+}{\circ}$ & $\begin{array}{l}\infty \\
\text { î } \\
\text { N. }\end{array}$ & $\begin{array}{l}\dot{\sigma} \\
\stackrel{\sigma}{2}\end{array}$ & $\begin{array}{l}\Rightarrow \\
\Rightarrow\end{array}$ \\
\hline & $\hat{\sim}$ & $\stackrel{N}{\sim}$ & $\overrightarrow{\hat{N}}$ & $\overrightarrow{\tilde{N}}$ & $\stackrel{2}{2}$ & $\begin{array}{l}N \\
\infty \\
\text { Na }\end{array}$ \\
\hline & $A$ & भे & 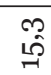 & $\begin{array}{l}\hat{d} \\
\dot{N}\end{array}$ & 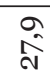 & $\begin{array}{l}\forall \\
\text { Q }\end{array}$ \\
\hline \multirow{3}{*}{ 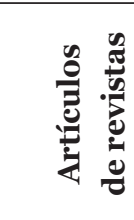 } & $\frac{\hat{z}}{\mathbf{z}}$ & $\stackrel{\theta}{\theta}$ & $\begin{array}{l}\hat{\sigma} \\
\hat{\text { N }}\end{array}$ & $\stackrel{+}{\infty}$ & $\stackrel{\circ}{\hat{\sigma}}$ & $\stackrel{+}{\stackrel{\sigma}{\sigma}}$ \\
\hline & $\hat{v}$ & $\hat{\approx}$ & $\stackrel{\Re}{\infty}$ & $\begin{array}{l}2 \\
\text { âे }\end{array}$ & $\overrightarrow{\widehat{N}}$ & $\vec{\infty}$ \\
\hline & $A$ & $\infty$ & $\hat{\sigma}$ & ஸิ & in & $\begin{array}{l}\text { ma } \\
\tilde{m}^{2}\end{array}$ \\
\hline \multirow{3}{*}{ 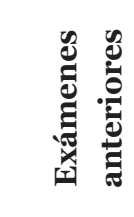 } & $\bar{z}$ & I & $m$ & $\infty$ & 0 & $\vec{\infty}$ \\
\hline & $\hat{\sim}$ & $\stackrel{+}{f}$ & ڤ̊ & $\begin{array}{l}0 \\
=\end{array}$ & $\underset{\sim}{0}$ & $\begin{array}{l}\text { N } \\
\text { LO }\end{array}$ \\
\hline & $A$ & $\begin{array}{l}\forall \\
\text { to }\end{array}$ & $\stackrel{\sigma}{\varrho}$ & $\begin{array}{l}\infty \\
\underset{f}{f}\end{array}$ & ลิ & کิ \\
\hline \multirow{3}{*}{ 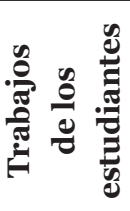 } & $\hat{\mathbf{z}}$ & $\stackrel{12}{\Lambda}$ & $\begin{array}{l}\hat{f} \\
\text { a }\end{array}$ & $\stackrel{\sigma}{\dot{J}}$ & 0 & 今̂ \\
\hline & $\tilde{\xi}$ & $\begin{array}{l}\infty \\
\stackrel{\rightarrow}{\sim}\end{array}$ & $\begin{array}{l}2 \\
\stackrel{2}{\sigma}\end{array}$ & $\begin{array}{l}+ \\
\text { ¿ }\end{array}$ & $\stackrel{N}{\infty}$ & $\begin{array}{l}12 \\
\mathscr{N}^{2}\end{array}$ \\
\hline & $A$ & $\begin{array}{l}0 \\
a\end{array}$ & $\begin{array}{l}12 \\
\hat{\lambda}\end{array}$ & $\begin{array}{l}\text { aे } \\
\text { d }\end{array}$ & $\begin{array}{l}12 \\
\infty D^{1}\end{array}$ & $\begin{array}{l}\text { N } \\
\text { J }\end{array}$ \\
\hline \multirow{3}{*}{ 晃 } & $\vec{z}$ & $\begin{array}{l}\infty \\
f \\
f\end{array}$ & $\begin{array}{l}+ \\
\stackrel{S}{N}\end{array}$ & $\hat{O}$ & $a$ & $m$ \\
\hline & $\frac{\theta}{2}$ & $\begin{array}{l}\infty \\
\hat{N} \\
\stackrel{N}{N}\end{array}$ & $\begin{array}{l}\text { a } \\
\text { în }\end{array}$ & $\begin{array}{l}\hat{N} \\
\hat{N}\end{array}$ & $\hat{\hat{N}}$ & ભે \\
\hline & A & $\stackrel{+}{\infty}$ & ลิ & $\begin{array}{l}\infty \\
\text { N̂}\end{array}$ & ลิ & $\underset{N}{\stackrel{J}{N}}$ \\
\hline \multirow{3}{*}{ 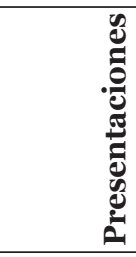 } & $\hat{\mathbf{z}}$ & $\stackrel{12}{\wedge}$ & $a$ & $a$ & $\begin{array}{l}a \\
\Rightarrow\end{array}$ & $\hat{\widehat{0}}$ \\
\hline & चे & เి & $\begin{array}{l}\infty \\
\hat{N}\end{array}$ & $\widehat{\curvearrowright}$ & $\hat{\Lambda}$ & $\stackrel{N}{N}$ \\
\hline & $A$ & $\begin{array}{l}\infty \\
\infty \\
\infty \\
1\end{array}$ & ป & $\hat{\sigma}$ & గొ & $\hat{\sigma}$ \\
\hline \multirow{4}{*}{$\frac{\underbrace{\mathscr{U}}}{3}$} & $\hat{\mathbf{z}}$ & $m$ & 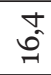 & ఫْ & $\begin{array}{l}\stackrel{\sigma}{0} \\
\text { ô }\end{array}$ & $\stackrel{2}{\stackrel{2}{n}}$ \\
\hline & $\tilde{\sigma}$ & $\begin{array}{c}N \\
\infty \\
\sim \\
N\end{array}$ & $\stackrel{\sim}{N}$ & $\begin{array}{l}+ \\
\text { ¿ }\end{array}$ & $\vec{\approx}$ & $\widehat{\curvearrowright}$ \\
\hline & $A$ & $\begin{array}{l}\hat{\sigma} \\
\hat{\sigma} \\
\text { No }\end{array}$ & $\begin{array}{l}\text { â } \\
\text { ô }\end{array}$ & ลิ & $\begin{array}{l}\mathfrak{\sigma}^{2} \\
\sigma^{2}\end{array}$ & $\begin{array}{l}+ \\
\text { L }\end{array}$ \\
\hline & & 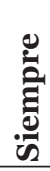 & 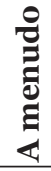 & 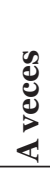 & 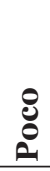 & 胥 \\
\hline
\end{tabular}


O. FLORES; I. DEL ARCO

INFLUENCIA DE LAS TIC EN LA UTILIZACIÓN DE MATERIALES Y RECURSOS EN LOS PROCESOS DE ENSEÑANZA-APRENDIZAJE...

\begin{tabular}{|c|c|c|c|c|c|c|}
\hline \multirow{3}{*}{ 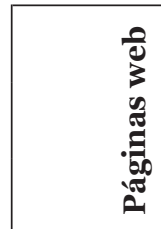 } & $\hat{z}$ & $\begin{array}{l}n \\
\dot{y} \\
\dot{y}\end{array}$ & $\begin{array}{l}\infty \\
\infty \\
\infty \\
\infty\end{array}$ & $\begin{array}{l}+ \\
\stackrel{0}{\circ}\end{array}$ & 1 & $\stackrel{12}{f}$ \\
\hline & $\tilde{\sigma}$ & $\begin{array}{l}10 \\
10 \\
10\end{array}$ & ํㅏ & $\begin{array}{l}\text { m } \\
\text { Nं }\end{array}$ & $\begin{array}{l}\infty \\
\hat{n} \\
\end{array}$ & $\begin{array}{l} \pm \\
\text { ó }\end{array}$ \\
\hline & $A$ & $\begin{array}{l}0 \\
\stackrel{0}{N}\end{array}$ & 0 & $\overrightarrow{\widehat{N}}$ & $\hat{\text { กิ }}$ & $\begin{array}{l}0 \\
\tilde{m}\end{array}$ \\
\hline \multirow{3}{*}{ 苞 } & $\vec{z}$ & $m$ & 0 & $m$ & $\stackrel{+}{+}$ & \begin{tabular}{l}
0 \\
\multirow{N}{N}{}
\end{tabular} \\
\hline & $\tilde{v}$ & $\begin{array}{l}\text { Ñ } \\
\text { N }\end{array}$ & $\begin{array}{l}\tilde{N} \\
\hat{\sim}\end{array}$ & $\stackrel{+}{+}$ & $\sigma \hat{\sigma}$ & $\hat{\sigma}$ \\
\hline & $A$ & $\begin{array}{l}\text { N } \\
\text { N̂ }\end{array}$ & $\mathrm{N}$ & $\stackrel{N}{N}$ & $\begin{array}{l}\infty \\
\hat{\sigma}^{-}\end{array}$ & $\hat{i}$ \\
\hline \multirow{3}{*}{ 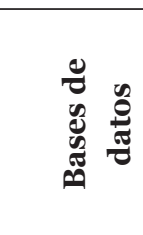 } & $\frac{\hat{z}}{\mathbf{z}}$ & $m$ & $\stackrel{+}{+}$ & $\begin{array}{l}\stackrel{\Omega}{2} \\
\approx\end{array}$ & $\stackrel{\curvearrowright}{\stackrel{\sim}{\sim}}$ & $\begin{array}{l}\infty \\
\dot{f}\end{array}$ \\
\hline & $\tilde{r}$ & ஸి & $\stackrel{N}{N}$ & $\begin{array}{c}N \\
\infty \\
\infty \\
-1\end{array}$ & 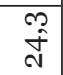 & $\begin{array}{l}\hat{\sigma} \\
\hat{s}\end{array}$ \\
\hline & $A$ & $\hat{\infty}$ & $\begin{array}{l}\hat{N} \\
\text { în }\end{array}$ & $\begin{array}{l}\infty \\
\stackrel{\infty}{\sim}\end{array}$ & $\begin{array}{l}\text { Na } \\
\text { î }\end{array}$ & $\overrightarrow{10}$ \\
\hline \multirow{3}{*}{ 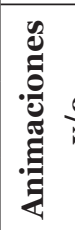 } & $\frac{\hat{z}}{\mathbf{z}}$ & 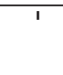 & $\begin{array}{l}a \\
\Rightarrow\end{array}$ & $\begin{array}{l}1 \rho \\
f\end{array}$ & $\begin{array}{l}+ \\
\stackrel{-}{*}\end{array}$ & $\hat{\widehat{\vartheta}}$ \\
\hline & $\hat{r}$ & लै & $\begin{array}{l}\infty \\
\infty \\
\infty \\
\end{array}$ & $\begin{array}{l}12 \\
0 \\
\tilde{N}^{2}\end{array}$ & $\overrightarrow{\widehat{A}}$ & 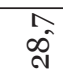 \\
\hline & A & $\stackrel{N}{\sim}$ & $\begin{array}{l}10 \\
\text { ஸి }\end{array}$ & $\begin{array}{l}0 \\
\text { న̂ }\end{array}$ & $\begin{array}{l}12 \\
\text { సి } \\
\text { N̂}\end{array}$ & $\begin{array}{l}\infty \\
\hat{1} \\
10\end{array}$ \\
\hline \multirow{3}{*}{ 串 } & $\frac{\hat{z}}{z}$ & $\stackrel{12}{1}$ & $\stackrel{+}{\circ}$ & $a$ & $\begin{array}{l}\hat{O} \\
\hat{\text { N}}\end{array}$ & $\hat{\circ}$ \\
\hline & $\bar{\omega}$ & $\hat{\text { ले }}$ & $\begin{array}{l}\text { N } \\
\text { N̂} \\
\mathcal{N}\end{array}$ & $\overrightarrow{\mathrm{N}}$ & $\begin{array}{l}\text { N } \\
\infty \\
\infty\end{array}$ & $\begin{array}{l}\text { N } \\
f\end{array}$ \\
\hline & $A$ & 1 & $\stackrel{12}{-1}$ & to & $\hat{\hat{A}}$ & $\overrightarrow{2}$ \\
\hline \multirow{3}{*}{ 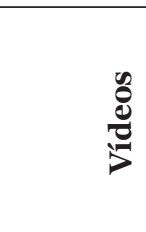 } & $\frac{\hat{z}}{z}$ & $\frac{10}{f}$ & 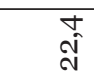 & $\hat{\dot{f}}$ & $\begin{array}{l}a \\
\vec{f}\end{array}$ & $\begin{array}{l}m \\
\tilde{o}^{2}\end{array}$ \\
\hline & $\hat{v}$ & $\hat{\curvearrowright}$ & $\begin{array}{l}\text { No } \\
\text { Nิ }\end{array}$ & $\hat{\sigma}$ & $\begin{array}{c}\text { No } \\
\infty \\
\underbrace{\prime}_{-1}\end{array}$ & $\begin{array}{l}\text { Oे } \\
\hat{\delta}\end{array}$ \\
\hline & $A$ & $\mathfrak{N}_{0}^{N}$ & $\stackrel{N}{f}$ & $\begin{array}{l}\text { N } \\
\vec{N}\end{array}$ & $\begin{array}{l}1 \Omega \\
\text { กิ }\end{array}$ & $\begin{array}{l}\forall \\
0 \\
0\end{array}$ \\
\hline \multirow{3}{*}{ 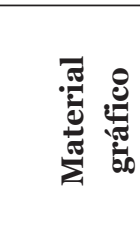 } & 安 & $\stackrel{12}{\wedge}$ & $\begin{array}{l}\infty \\
\vec{\sigma}\end{array}$ & $\begin{array}{l}+ \\
\stackrel{\sigma}{2}\end{array}$ & $\begin{array}{l}\text { a } \\
\dot{J}\end{array}$ & ఫ্] \\
\hline & ซे & $\begin{array}{l}12 \\
\hat{4}\end{array}$ & $\begin{array}{l}\infty \\
\text { ᄋे }\end{array}$ & $\begin{array}{l}\text { బิ } \\
\text { సे }\end{array}$ & $\begin{array}{l}\text { NV } \\
\text { N̂}\end{array}$ & 10 \\
\hline & A & $\vec{F}$ & $\begin{array}{l}\hat{\sigma} \\
\hat{N}\end{array}$ & $\begin{array}{l}\overrightarrow{0} \\
\hat{m}\end{array}$ & ㅇ & $\stackrel{+}{\circ}$ \\
\hline & & 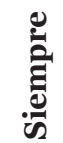 & 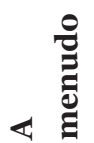 & 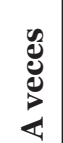 & : & $\underset{\mathrm{Z}}{\mathrm{Z}}$ \\
\hline
\end{tabular}

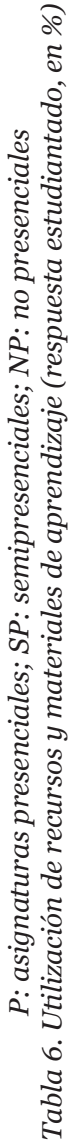


En referencia a los resultados agrupados por género, estos no mostraron diferencias significativas entre hombres y mujeres ni por parte del profesorado ni del estudiantado. Es interesante mencionar el hecho de que se detectó una diferencia significativa en el uso del vídeo, observando que era una estrategia más utilizada por hombres que por mujeres, como se muestra en la tabla 7.

\begin{tabular}{|l|c|c|}
\hline \multicolumn{1}{|c|}{ VIDEOS } & Hombre & Mujer \\
\hline Siempre & 11,6 & 3,6 \\
\hline A menudo & 18,6 & 7,1 \\
\hline A veces & 34,9 & 28,6 \\
\hline Poco & 11,6 & 21,4 \\
\hline Nunca & 23,3 & 39,3 \\
\hline
\end{tabular}

Tabla 7. Utilización de vídeo según género profesorado (en \%)

Finalmente, los resultados agrupados por experiencia nos mostraron que la experiencia docente no era un elemento discriminatorio a la hora de utilizar diferentes recursos y materiales de aprendizaje. Los datos en general se comportaron de manera similar en todas las franjas de edad, aunque se observaron pequeñas diferencias en algunos aspectos como que los artículos de revistas, los vídeos o las animaciones no eran recursos muy utilizados por el profesorado más veterano, como puede observarse en las tablas 8,9 y 10 respectivamente.

\begin{tabular}{|l|c|c|c|c|}
\hline \multirow{2}{*}{$\begin{array}{c}\text { ARTÍCULOS DE } \\
\text { REVISTAS }\end{array}$} & \multicolumn{4}{|c|}{ AÑOS EXPERIENCIA } \\
\cline { 2 - 5 } & Menos de 5 & $\begin{array}{c}\text { De 5 a } \\
\mathbf{1 0}\end{array}$ & $\begin{array}{c}\text { De 11 a } \\
\mathbf{2 0}\end{array}$ & Más de 2o \\
\hline Siempre & - & 18,2 & 20 & 10 \\
\hline A menudo & 50 & 36,4 & 36,7 & $5,0-$ \\
\hline A veces & 20 & 9,1 & 16,7 & 35 \\
\hline Poco & 10 & 27,3 & $10,0-$ & 45 \\
\hline Nunca & - & 9,1 & 16,7 & - \\
\hline NS / NC & 20 & - & - & 5 \\
\hline
\end{tabular}

Tabla 8. Utilización de artículos de revistas según experiencia profesorado (en \%) 


\begin{tabular}{|l|c|c|c|c|}
\hline \multirow{2}{*}{ VÍDEOS } & \multicolumn{4}{|c|}{ AÑOS EXPERIENCIA } \\
\cline { 2 - 5 } & Menos de 5 & De 5 a 10 & De 11 a 2o & Más de 20 \\
\hline Siempre & 10 & $\mathbf{1 8 , 2}$ & 6,7 & 5 \\
\hline Menudo & 30 & 18,2 & 6,7 & 15 \\
\hline A veces & 30 & 45,5 & 30 & 30 \\
\hline Poco & - & 9,1 & 26,7 & 10 \\
\hline Nunca & 30 & 9,1 & 30 & 40 \\
\hline
\end{tabular}

Tabla 9. Utilización de vídeos según experiencia profesorado (en \%)

\begin{tabular}{|c|c|c|c|c|}
\hline \multirow{2}{*}{$\begin{array}{l}\text { ANIMACIONES Y / O } \\
\text { SIMULACIONES }\end{array}$} & \multicolumn{4}{|c|}{ AÑOS EXPERIENCIA } \\
\hline & $\begin{array}{c}\text { Menos } \\
\text { de } 5\end{array}$ & $\begin{array}{c}\text { De } 5 \text { a } \\
10 \\
\end{array}$ & $\begin{array}{c}\text { De } 11 \text { a } \\
20\end{array}$ & Más de 20 \\
\hline Siempre & 20 & - & 10 & - \\
\hline A menudo & - & 18,2 & 3,3 & 5 \\
\hline A veces & 30 & 18,2 & 13,3 & 5 \\
\hline Poco & 10 & 18,2 & 23,3 & 15 \\
\hline Nunca & 30 & 45,5 & 46,7 & 60 \\
\hline NS / NC & 10 & - & 3,3 & 15 \\
\hline
\end{tabular}

Tabla 10. Utilización de animaciones y/o simulaciones según experiencia profesorado (en \%)

Sobre el estudiantado, los de primer año indicaron que en las clases se utilizaban más las presentaciones a través de diapositivas que el resto, mientras que se observó que, según el estudiantado, en los últimos cursos aumentaba la utilización de artículos de revistas, como se puede observar en las tablas 11 y 12 respectivamente.

\begin{tabular}{|l|c|c|c|c|}
\hline $\begin{array}{c}\text { PRESENTACIONES A } \\
\text { TRAVÉS DE DIAPOSITIVAS } \\
\text { DE CLASE }\end{array}$ & \multicolumn{4}{|c|}{ AÑOS EN LA UNIVERSIDAD } \\
\hline & $\begin{array}{c}\text { Primer } \\
\text { año }\end{array}$ & $\begin{array}{c}\mathbf{2} / \mathbf{3} \\
\text { años }\end{array}$ & $\begin{array}{c}\mathbf{4} / \mathbf{5} \\
\text { años }\end{array}$ & $\begin{array}{c}\text { Más de 5 } \\
\text { años }\end{array}$ \\
\hline Siempre & $64,3+$ & $39,8-$ & $15,7-$ & $21,1-$ \\
\hline A menudo & $16,9-$ & $32,5+$ & $35,3+$ & 21,1 \\
\hline A veces & 7 & 6,6 & 11,8 & 7,9 \\
\hline Poco & 4,9 & 4,2 & 2 & 18,4 \\
\hline Nunca & $6,3^{-}$ & 16,9 & $35,3+$ & 31,6 \\
\hline
\end{tabular}

Tabla 11. Utilización de presentaciones según años en la universidad estudiantado (en \%) 


\begin{tabular}{|l|c|c|c|c|}
\hline \multirow{2}{*}{$\begin{array}{l}\text { ARTÍCULOS DEVISTAS } \\
\text { RE }\end{array}$} & \multicolumn{4}{|c|}{ AÑOS EN LA UNIVERSIDAD } \\
\cline { 2 - 5 } & Primer año & $\begin{array}{c}\mathbf{2} / \mathbf{3} \\
\text { años }\end{array}$ & $\begin{array}{c}\mathbf{4} / \mathbf{5} \\
\text { años }\end{array}$ & $\begin{array}{c}\text { Más de 5 } \\
\text { años }\end{array}$ \\
\hline Siempre & 2,9 & 5,4 & 2 & 5,3 \\
\hline menudo & 7,3 & 7,2 & 17,6 & 21,1 \\
\hline A veces & 23,4 & $32,5+$ & 21,6 & 31,6 \\
\hline Poco & 25,3 & 30,1 & 23,5 & 21,1 \\
\hline Nunca & $40.9+$ & 23,5 & 35,3 & 21,1 \\
\hline
\end{tabular}

Tabla 12. Utilización de presentaciones según años en la universidad estudiantado (en \%)

\section{Entrevistas}

Las entrevistas nos sirvieron para verificar, entre otras cosas, que la utilización de recursos en formato digital era una estrategia genérica independientemente de la modalidad formativa de la asignatura. En las asignaturas presenciales aparecía el PowerPoint como una herramienta unánime para apoyar las exposiciones orales de los contenidos. Además, aparte de los libros y las partes de libros, los artículos en formato electrónico, las páginas web, ficheros con problemas resueltos, vídeos... estaban presentes como recursos en estas asignaturas. En cuanto a los vídeos, se observó como el profesorado de las asignaturas presenciales mostraban preocupación por la duración de estos e indicaba que hay que utilizar vídeos breves: “deben ser siempre vídeos muy cortos, pequeños detalles, porque con vídeo largo, incluso los alumnos, desconectan, y es absolutamente inoperante" (Profesor del ámbito de ingeniería y arquitectura, cuatro años de experiencia docente en la universidad). Otra característica interesante es que en las asignaturas presenciales se utilizaba el campus virtual para hacer llegar estos recursos a los estudiantes.

También en las entrevistas realizadas al profesorado de las asignaturas semipresenciales se detectó la utilización de una variedad considerable de recursos, tanto en formato papel (apuntes, partes de libros, artículos de revistas o de prensa) como en formato digital (páginas Web, vídeos, audio, imágenes o ficheros electrónicos con problemas resueltos). Los recursos en formato digital se ponían a disposición de los estudiantes a través del campus virtual.

Finalmente, en las asignaturas no presenciales también se observó que la variedad de recursos que se ofrecían al estudiantado era bastante amplia. Partiendo de una información básica, el profesorado ofrecía una variedad de material considerable en diferentes formatos: esquemas y resúmenes de lo más importante, transparencias, 
artículos, conferencias en vídeo, libros o partes de libros, documentales, películas, noticias de actualidad relacionadas con la asignatura...

\section{DISCUSIÓN}

Nuestro estudio ha mostrado que, independientemente dela modalidad formativa de las asignaturas, se utiliza una variedad considerable de recursos para hacer llegar la información a los estudiantes. Por un lado, tenemos lo que en el ámbito coloquial se denomina apuntes de la asignatura, que el profesor sigue proporcionando a los estudiantes oralmente (y casi siempre con el apoyo de programas de presentación tipo PowerPoint) o en formato electrónico, complementados con una serie de materiales como artículos, vídeos, páginas Web, partes de libros...

Tanto profesorado como estudiantes consideran que los contenidos son un pilar importante del proceso formativo y así lo valoran, y en este aspecto coincidimos con otros estudios analizados como los de Chen (2007) y Husnayati, Bunyarit y Hussein (2009). Hemos detectado que el profesorado se muestra muy detallista a la hora de indicar la cantidad de recursos y de materiales que pone a disposición del estudiantado, lo que nos puede llevar a preguntarnos si realmente los estudiantes aprovechan toda la información que se les facilita y hasta qué punto hay una mediación entre los materiales que publica el profesorado y el acceso que hacen los alumnos, como mencionábamos con las ideas de Gros y Kirschner (2006). También en este sentido podríamos preguntarnos aspectos que nos indica Wright (2003) sobre cómo se presentan los diferentes recursos y materiales que se ponen a disposición del estudiantado y si realmente estos son apropiados para lo que se pretende conseguir o no.

Otro aspecto observado es la tendencia a aumentar la variedad de recursos para utilizar a medida que aumenta la no presencialidad de las asignaturas. Nuestra conclusión sobre este hecho es que probablemente la disminución de las clases presenciales genera en el profesorado la necesidad de ofrecer más variedad de recursos para facilitar la asimilación de conceptos. Esta es una idea interesante, en la que se podría profundizar en otros estudios.

Desde nuestro punto de vista, aún queda mucho camino por recorrer y todavía se debe profundizar más en la utilización de recursos y materiales en los procesos de enseñanza-aprendizaje online. Como hemos comentado, descubrir los usos que los estudiantes hacen de los materiales que el profesorado pone a su disposición es una propuesta que nuestro trabajo no ha podido recoger. También, conocer hasta 
qué punto existe una mediación entre los materiales que publica el profesorado y el acceso que hacen los alumnos, y en qué medida este hecho supone que estos sean consultados o no puede ser otra línea de futuro. Otros posibles proyectos pueden ir en la línea de descubrir por qué existe la tendencia a aumentar la variedad de recursos que se ponen a disposición de los estudiantes a medida que aumenta la no presencialidad de las asignaturas, aspecto que hemos podido detectar en nuestro trabajo.

\section{REFERENCIAS BIBLIOGRÁFICAS}

Baelo, R. (2009). El e-learning, una respuesta educativa a las demandas de las sociedades del siglo XXI. PixelBit. Revista de Medios y Educación, 35, (87-96). [en línea] Disponible en: http://www.sav.us.es/pixelbit/ pixelbit/articulos/n35/7.pdf (consulta 2011, 15 de marzo).

Chen, S. (2007). Instructional Design Strategies for Intensive Online Courses: An Objectivist-Constructivist Blended Approach. Journal of Interactive Online Learning, 6 (1), (72-86). [en línea] Disponible en: http://www.ncolr. org/jiol/issues/PDF/6.1.6.pdf (consulta 2009, 23 de diciembre).

Escofet, A.; Alabart, A.; Vilà, G. (2006). Ensenyar $i$ aprendre amb TIC a la universitat. Barcelona: ICE de la Universitat de Barcelona. [en línea] Disponible en: http://www.ub.es/ forum/Archivos/escofet.pdf (consulta 2011, 14 de marzo).

Gros, B.; Kirschner, P. (2006). La recerca sobre la docència a la universitat: l'ús d'entorns electrònics en l'educació superior. Barcelona: Institut de Ciències de l'Educació de la Universitat de Barcelona. [en línea] Disponible en: http://www.ub.es/forum/Archivos/ kirschnergros.pdf (consulta 2011, 17 de marzo).

Hernández Pina, F. (1995). Bases metodológicas de la investigación educativa. Fundamentos. Murcia: Promociones y Publicaciones Universitarias, SA.

Izquierdo, R.; María, R. (2010). El impacto de las TIC en la transformación de la enseñanza universitaria: repensar los modelos de enseñanza y aprendizaje. Teoría de la Educación. Educación y Cultura en la Sociedad de la Información, 11 (1), (32-68).

Latorre, A.; Del Rincón, D.; Arnal, J. (2003). Bases metodológicas de la investigación educativa. Barcelona: Experiencia.

Mahdizadeh, H.; Biemans, H.; Mulder, M. (2008). Determining factors of the use of e-learning environments by University teachers. Computers \& Education, 51, (142-154).

Marín, V.; Romero, M. A. (2009). La formación docente universitaria a través de las TICs. Pixel-Bit. Revista de Medios y Educación, 35, (97-103). [en línea] Disponible en: http://www. sav.us.es/pixelbit/pixelbit/articulos/ n35/8.pdf (consulta 2011, 16 de marzo).

Monge, S. (2007). La escuela vasca ante el cambio tecnológico. Tecnologías de la Información y la Comunicación en la enseñanza (1999-2004). [en línea] Disponible en: http://www. sergiomonge.com/doc/tesis-doctoralsergio-monge.pdf (consulta 2009, 12 de noviembre). 
Mülbert, A.; Matuzawa, F. (2011). La transformación del proceso de diseño institucional: del impreso a la multimedia. RIED. Revista Iberoamericana de Educación, 14 (1), (41-54). [en línea] Disponible en: $\quad$ http://www.utpl.edu.ec/ ried/images/pdfs/volumen141/ latransformaciondelproceso.pdf (consulta 2011, 20 de octubre).

Pedró, F.; Benavides, F. (2007). Políticas educativas sobre nuevas tecnologías en los países iberoamericanos. RIE. Revista Iberoamericana de Educación, 45, (19-70). [en línea] Disponible en: http://www.rieoei.org/rie45a01.pdf (consulta 2011, 14 de marzo).
Salinas, J. (2004). Innovación docente y uso de las TIC en la enseñanza universitaria. Revista Universidad y Sociedad de Conocimiento, 1 (1), (1-16). [en línea] Disponible en: http://www.uoc.edu/ rusc/dt/esp/salinas1104.pdf (consulta 2011, 14 de marzo).

Wright, C. R. (2003). Criteria for Evaluating the Quality of Online Courses. Edmonton: Grant MacEwan College. [en línea] Disponible en: http://elearning.typepad.com/ thelearnedman/ID/evaluatingcourses. pdf (consulta 2011, 17 de marzo).

\section{PERFIL ACADÉMICO Y PROFESIONAL DE LOS AUTORES}

Óscar Flores Alarcia. Doctor en Psicopedagogía. Coordinador del Área de Soporte a la Innovación Docente y E-learning del ICE-CFC de la UdL. Profesor asociado a tiempo parcial en la Facultad de Ciencias de la Educación de la UdL. Las principales líneas de trabajo se relacionan con los procesos formativos a través de la tecnología (formación semipresencial y no presencial) y también la realización de diferentes estudios sobre elementos del proceso de enseñanza-aprendizaje (la innovación docente las competencias, la organización, la evaluación, etc.).

E-mail: oscar.flores@udl.cat

Isabel del Arco Bravo. Doctora en Psicopedagogía con Premio extraordinario de doctorado por la Universidad de Lleida. Profesora Titular de Universidad del Departamento de Pedagogía y Psicología de la UdL. Su actividad profesional se ha desarrollado preferentemente en los diferentes niveles y etapas educativas del sistema público de enseñanza como funcionaria del cuerpo del profesorado. Las líneas de investigación han sido preferentemente las relativas a la atención a la diversidad desde el educativo y como reorientar el proceso de enseñanza/aprendizaje. Temas relativos a la Educación Intercultural, la figura del educador familiar, intervención de profesores de apoyo en el aula y la innovación en docencia universitaria. Ha sido Directora del ICE de la UdL hasta el 2005, hasta que pasó a ser Vicerrectora de Docencia, cargo que ocupó hasta mayo de 2011.

E-mail: del.arco@pip.udl.cat 
DIRECCIÓN DE LOS AUTORES

Universidad de Lleida

Facultad de Ciencias de la Educación

C/ Jaume II, 71

25001, Lleida, España.

Fecha de recepción del artículo: 20/10/11

Fecha de aceptación del artículo: 05/02/12

\section{Como citar este artículo:}

Flores Alarcia, O.; del Arco Bravo, I. (2012). Influencia de las TIC en la utilización de materiales y recursos en los procesos de enseñanza-aprendizaje de la Universidad de Lleida: ¿uso o abuso? RIED. Revista Iberoamericana de Educación a Distancia, volumen $15, \mathrm{n}^{0} 2$, pp. 191-213. 